\title{
Operational limits on WEST inertial divertor sector during the early phase experiment
}

\author{
M. Firdaouss ${ }^{a}$, Y. Corre ${ }^{a}$, P. Languille ${ }^{a}$, H. Greuner ${ }^{b}$, E. Autissier $^{a}$, C. Desgranges ${ }^{a}$, D. Guilhem ${ }^{a}$, J. \\ P. Gunn ${ }^{\mathrm{a}}$, M. Lipa ${ }^{\mathrm{a}}$, M. Missirlian ${ }^{\mathrm{a}}$, J.-Y. Pascal ${ }^{\mathrm{a}}$, C. Pocheau ${ }^{\mathrm{a}}$, M. Richou ${ }^{\mathrm{a}}$, E. Tsitrone ${ }^{\mathrm{a}}$
}

\author{
a CEA, IRFM, F-13108 Saint-Paul-Lez-Durance, France \\ b Max-Planck-Institut for Plasma Physics, Boltzmannstr. 2, 85748 Garching, Germany
}

E-mail: mehdi.firdaouss@cea.fr

\begin{abstract}
The primary goal of the WEST project is to be a test-bed to characterize fatigue and lifetime of ITER-like W divertor components subjected to relevant thermal loads. During the first phase of exploitation (S2 2016), these components (W monoblock plasma facing unit - W-PFU) will be installed in conjunction with graphite components (G-PFU). As the G-PFU will not be actively cooled, it is necessary to ensure that the foreseen pulse duration allows for the W PFU to reach their steady-state without overheating the G-PFU assembly structure or the embedded stainless steel diagnostics. High heat flux test have been performed at the GLADIS facility to assess the thermal behaviour of the G-PFU. Finally, some operational limit depending on plasma parameters are determined. It is shown that it is possible to operate at an injected power such that the maximal incident heat flux on the lower divertor is $10 \mathrm{MW} / \mathrm{m}^{2}$ for the required pulse length.
\end{abstract}

\section{Introduction}

The main objective of the WEST (W Environment in Steady-state Tokamak) project, which is an upgrade of the Tore Supra limiter machine, is to fabricate and test an ITER-like actively cooled tungsten divertor made of $456 \mathrm{~W}-\mathrm{PFU}$, in order to reduce the manufacturing and divertor operation risks for ITER. Therefore the magnetic $\mathrm{X}$-point configuration (lower single null, upper single null or double null) will be created by the installation of divertor coils inside the vacuum vessel. Plasma facing components (PFC) comprising the upper divertor and baffle are W-coated actively cooled components with a CuCrZr based heat sink. The W-PFU should withstand heat fluxes close to $10 \mathrm{MW} / \mathrm{m}^{2}$, which is expected during ITER normal operation and even $20 \mathrm{MW} / \mathrm{m}^{2}$ during slow transients [1]. It is therefore necessary to verify that for those levels of power the remaining PFCs are not overloaded.

Three different designs of the lower divertor target, composed of 12 sectors of 38 PFU each are already foreseen because of delays in production of the W-PFU. The first version to be installed will be made of nonactively cooled graphite components with a thin W coating $(<15 \mu \mathrm{m})$. The second version will be made of actively cooled CuCrZr components also with thin $\mathrm{W}$ coatings. Then it will be progressively replaced by the third version made of W monoblocks bonded to a copper alloy tube (ITER-like W-PFU). It is important to note that all those three types of PFU will have an identical surface exposed to the plasma, meaning that the same scenario will produce the same heat flux on any of them. The difference will be in the capability of the G-PFU to endure a high level heat load during long pulses.

The goal of this paper is to analyse the operational limitations attributed to the graphite inertial sectors and discuss the consequences for the scientific exploitation of WEST during the early operation phase. The operational limitations on this PFU are due to the combination of different kinds of issues: damage of the G-PFU attachment to the support, melting of the embedded stainless steel diagnostics and delamination of the coating interface. The first two cases were carefully analysed during the design phase with finite elements simulations, which is reported in the first section. The assumptions made during the design phase were tested with dedicated high heat flux experiments at GLADIS neutral beam facility [2]. These results are presented in the second 
section. Finally, we discuss the coating limits (already known from past experiments) compared to the limits defined in the previous section. The main limit depends on the scenario: for high power/short pulses, the limit is driven by the coating (due to the high surface temperature) while for low power/long pulses it is driven by the stainless steel diagnostics (due to diffusion of heat into the bulk of the PFU). Nevertheless it is possible to operate with a maximal incident heat flux of $10 \mathrm{MW} / \mathrm{m}^{2}$ on the lower divertor during the required duration without risking the integrity of the G PFU.

\section{Mechanical attachment optimization and localization definition of the embedded diagnostics}

As mentioned previously the G-PFUs installed on the lower divertor during the first exploitation phase are made of graphite. 13 different types of G-PFU are planned. They are then assembled in $30^{\circ}$ sectors of 38 (standard sector) or 36 (sector with Langmuir probes) G-PFU. Each individual G-PFU is poloidally divided in two parts, high field side (HFS) and the low field side (LFS) PFU (see Fig. 1). The gap between those two GPFU is in the private flux region below the X-point for the foreseen plasma configuration ([3]). Both of LFS and HFS PFU are shaped. The other types of G-PFU are mainly equipped with embedded diagnostics such as thermocouples (TC - up to $1100^{\circ} \mathrm{C}$ ) and fiber Bragg grating (FBG - up to $1200^{\circ} \mathrm{C}$ ) for measuring the temperature and Langmuir Probes (LP) for measuring some plasma physical properties. The PFU with LP are 1.5 times larger than the standard elements, to provide sufficient volume to house the probes without weakening the G$\mathrm{PFU}$, and space to lay the 58 coaxial cables used to power the probes.

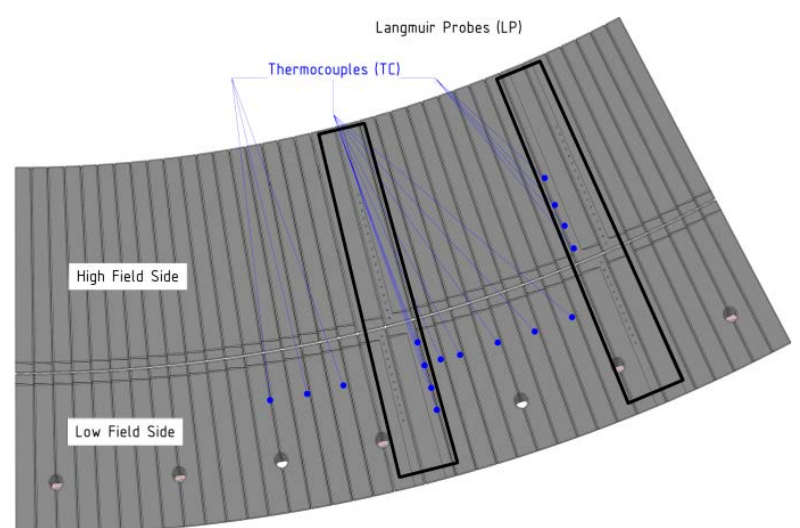

Full $30^{\circ}$ sector equipped with diagnostics

Figure 1: $30^{\circ}$ sector of 36 PFU equipped with 58 Langmuir Probes and 16 thermocouples

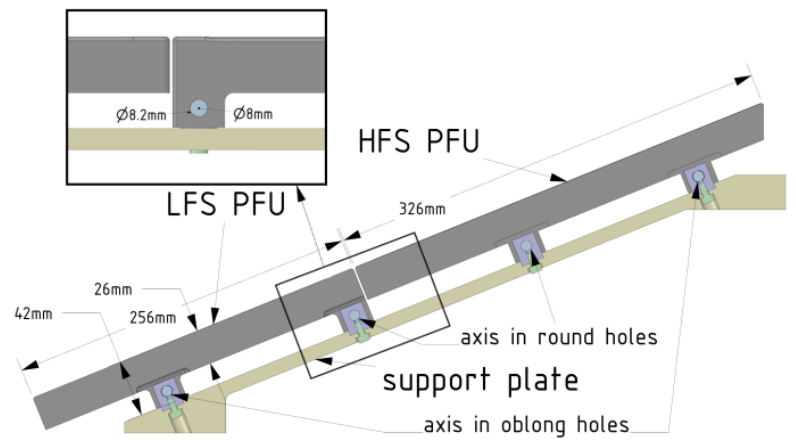

Figure 2: Side view of the LFS and HFS PFU with their attachment to the support plate

The material used for the best compatibility in terms of coefficient of thermal expansion with the $\mathrm{W}$ coating is the graphite R6710 from SGL, the same as used in the ASDEX Upgrade divertor [4, 5]. However this material exhibits a brittle behaviour and has a low ultimate strength, typically $170 \mathrm{MPa}$ for a compressive stress and $50 \mathrm{MPa}$ for a tensile stress. For that reason the G-PFU are linked to their support with a mobile attachment (see Fig. 2): on one extremity the hole containing the pin is round; on the other it is oblong to accommodate thermal expansion. However it is necessary to ensure that the stress in this area is below the design limit. 
The limitations originating from the coating are well known, since the same coating has been used for JET ITER-like Wall and ASDEX Upgrade $[4,5]$. In general, the W coating (including a Mo inter-layer) has shown its capability to sustain temperature up to $1800^{\circ} \mathrm{C}$ for at least 100 cycles without delamination. However, this conclusion depends greatly of the total heating time. Indeed, it is clearly shown [5] that the carbidisation of the Mo and $\mathrm{W}$ which occurs above $1200^{\circ} \mathrm{C}$, greatly weakens the coating. Therefore it is foreseen to limit the surface temperature of the G-PFU to $1200^{\circ} \mathrm{C}$ for the standard operation, but to allow to go further for a limited number of dedicated experiment, for example up to $1600^{\circ} \mathrm{C}$ to $1800^{\circ} \mathrm{C}$.

\subsection{Stress at the attachment interface}

In a first design stage, the bore for the axis in the graphite is $8 \mathrm{~mm}$, the same size than the axis. Finite element simulations have been performed with the ANSYS code. The thermal load is chosen in the PFCFluxcode simulations [3] from available assumptions to maximize the thermal energy and therefore the mechanical stress: $5 \mathrm{MW} / \mathrm{m}^{2}$ (peaked) during $15 \mathrm{~s}$, followed by 1000 s of cooling by radiation in close X-point plasma configuration with a decay length (lq) of $5 \mathrm{~mm}$ (important spreading of the heat flux).

The maximal surface temperature after $15 \mathrm{~s}$ is $1700^{\circ} \mathrm{C}$. After $1000 \mathrm{~s}$ for the cooling phase, the temperature is homogeneous in the PFU at $250^{\circ} \mathrm{C}$. The maximal tensile stress has been calculated during both heating and cooling phases, especially near the attachment axis. The size of the hole is optimized in order to minimize the tensile stress, which has to be lower than 13MPa (ultimate tensile strength of 50MPa with a margin ratio of 4).

\begin{tabular}{l|cc|cc}
\hline $\begin{array}{l}\text { Max tensile } \\
\text { stress (MPa) }\end{array}$ & \multicolumn{2}{|c|}{ 15s } & \multicolumn{2}{c}{ 1000s } \\
\hline$\varnothing$ hole & Axis & Bulk & Axis & Bulk \\
\hline $8 \mathrm{~mm}$ & 30 & 40 & 80 & 15 \\
$8.1 \mathrm{~mm}$ & 2 & 15 & 10 & 0.5 \\
$8.2 \mathrm{~mm}$ & 1 & 12 & 5 & 0.2 \\
\hline
\end{tabular}

Table 1: Evolution of the calculated maximal tensile stress in the PFU at the end of heating (15s) and cooling (1000s) phases, for different hole diameters, near the attachment axis and in the bulk material.

It is clear from the results presented in the table 1 that holes with dimensions too close to the nominal diameter of the axis could be very damageable to the PFU. The chosen diameter is $8.2 \mathrm{~mm}$, which allows a compatible tensile strength while ensuring a reasonable attachment clearance.

\subsection{Temperature field near the diagnostics.}

Under the same conditions, the position of the diagnostics has been verified in order to ensure an acceptable temperature range for the instrumentation. The TC are planned to be installed 7.5mm underneath the incident surface, the FBG $4 \mathrm{~mm}$ and $7.5 \mathrm{~mm}$. The screwing support of the LP will be located $20 \mathrm{~mm}$ below the surface.

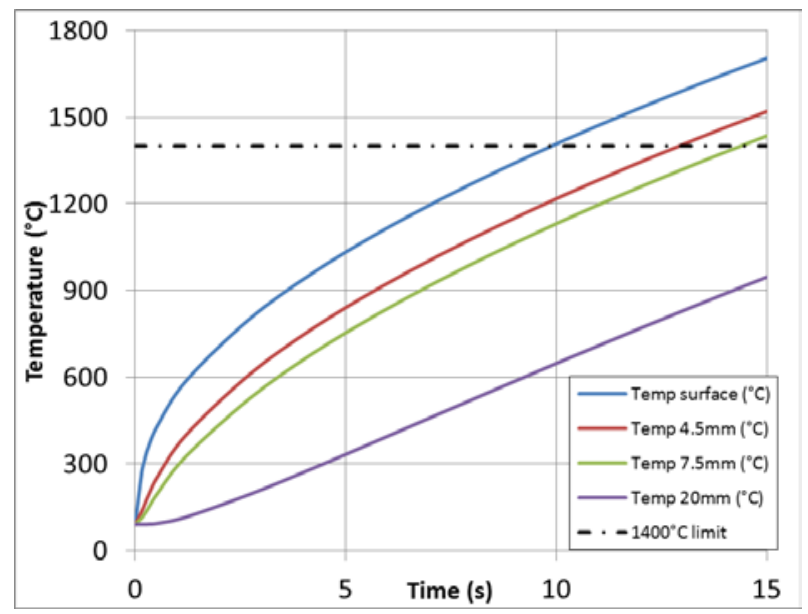

Figure 3: Temperature in the PFU at different depth as a function of time for $5 \mathrm{MW} / \mathrm{m}^{2}$ during $15 \mathrm{~s}$ 
The initial design of the LP fixation scheme and the sheaths of the TC and FBG are in stainless steel, whose melting point is around $1400^{\circ} \mathrm{C}$ (dashed line on figure 3). From figure 3 it is clear that at the end of the heat flux pulse the graphite near the TC and FBG will be at a higher temperature than the melting point of the steel for this conservative case. However, the melting of the sheaths would suppose a perfect thermal conductivity, which is not the case. Therefore it can be assumed that the TC and FBG won't be severely damaged during operations. Finally, the fixation system of the LP is far enough below the surface, and the maximal temperature around it is $900^{\circ} \mathrm{C}$.

\section{High heat flux test}

In order to evaluate the thermomechanical behaviour of the G-PFU and the endurance of , HHF tests have been performed on this inertial component. During these tests, the attachment integrity should be checked to verify that it can tolerate the deformations caused by the temperature field, both during the heating phase (peaked temperature) and after the cooling phase (homogeneous temperature) as shown in the previous section. The tested component was composed of two different types of elements, one was the standard type and the second the larger one (see previous section). Also two prototypes of LP and one TC were installed just under the neutral beam maximal heat flux pattern.

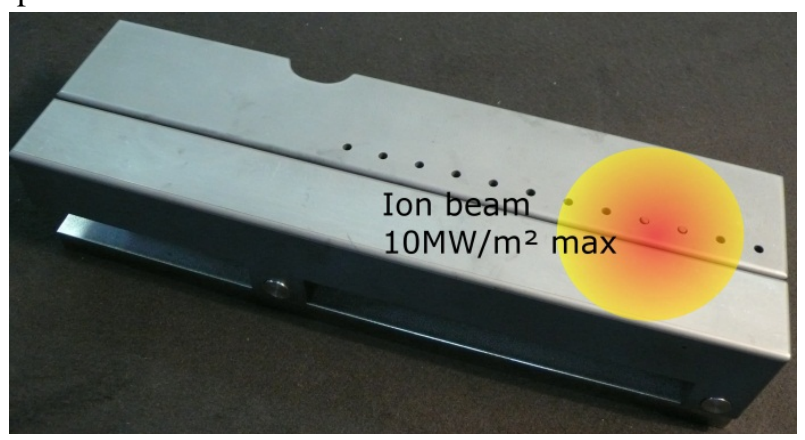

Figure 4: Assembly of the two tested PFU

The following loadings have been completed: 1 pulse at $3.5 \mathrm{MW} / \mathrm{m}^{2}$ during $5 \mathrm{~s}$ ( $\mathrm{Tmax}=620^{\circ} \mathrm{C}$ ), 2 pulses at $3.5 \mathrm{MW} / \mathrm{m}^{2}$ during $18 \mathrm{~s}\left(\operatorname{Tmax}=1200^{\circ} \mathrm{C}\right), 2$ pulses at $10.5 \mathrm{MW} / \mathrm{m}^{2}$ during $3.5 \mathrm{~s}\left(\mathrm{Tmax}=1600^{\circ} \mathrm{C}\right)$ and 1 pulse at $3.5 \mathrm{MW} / \mathrm{m}^{2}$ during $40 \mathrm{~s}$ ( $\operatorname{Tmax}=1600^{\circ} \mathrm{C}$ ). ANSYS simulations were done with parameters similar (heat flux and duration) than those used during the HHF tests. The surface temperature was measured by an infrared pyrometer at GLADIS. It was compared to the temperature computed on Figure 5. For both extreme pulses $\left(3.5 \mathrm{MW} / \mathrm{m}^{2}-\right.$ $40 \mathrm{~s}$ and $\left.10 \mathrm{MW} / \mathrm{m}^{2}-3.5 \mathrm{~s}\right)$, the temperature development was well simulated. It confirms that modelling was appropriate to describe this object.

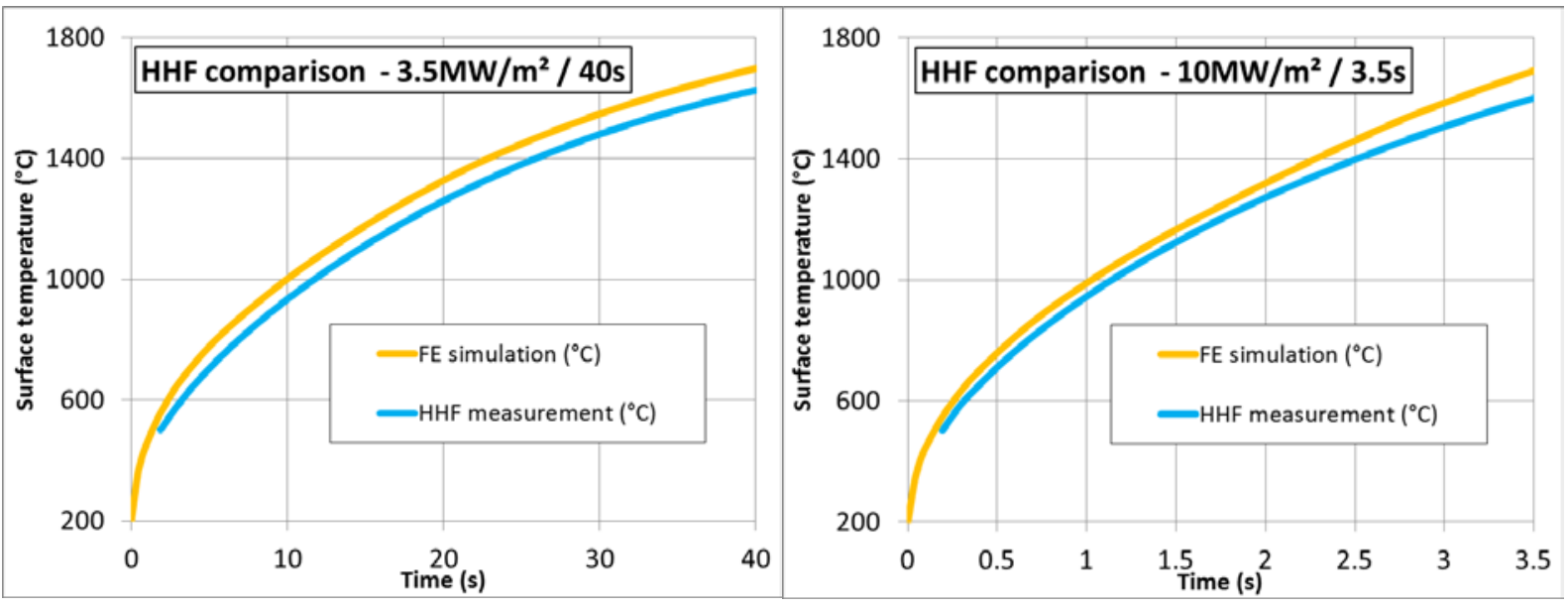

Figure 5: Comparison of the surface temperature measured during HHF tests with ANSYS simulations

The test was positive, as no cracks occurred for the different load cases. In particular, the attachment had proven its ability to sustain the high temperature due to a low heat flux for longer pulse duration. The TC did not 
break during the test. Contrary to the FEM prediction (section 2.2), the LP inserted in the G-PFU did not survive the HHF test. Indeed, the steel of the screwing support has partially melted, and it was impossible to unscrew. This was unexpected from the previous simulations. Actually two details have been neglected. The first is that the heat flux coming onto the head of the LP is mainly conducted through the LP itself, because LP and G-PFU have poor thermal contact. The second is that some heat flux may be transferred to the LP by thermal radiation between the graphite and the steel surface. Therefore it is plausible that the temperature of the LP was in fact higher than the temperature of the G-PFU, and high enough to melt partially the steel part of the LP.

\section{Operational limitations}

It can be concluded from the HHF experiment that ANSYS simulations produce valid results in terms of temperature, except for the steel LP diagnostics because of uncertainty in their thermal loads and thermal contact. In order to provide safer margins, and to avoid introducing an operational limit imposed by a diagnostic for the extremely energetic discharge, it has been decided to replace all LP stainless steel components by tantalum.

Therefore, except for this particular diagnostic, it is possible to plot the temperature at the surface and $7.5 \mathrm{~mm}$ below the surface for different plasma parameters and understand where the limit comes from: either from the coating $\left(1200^{\circ} \mathrm{C}\right.$ or $\left.1700^{\circ} \mathrm{C}\right)$ or from the diagnostic $\left(1000^{\circ} \mathrm{C}\right.$ to keep some margins to steel melting point). As already said in the first section, the most limiting case, from the thermal point of view, is the one with the maximal heat flux spreading. Therefore the same case is kept: close X-point with a decay length of $5 \mathrm{~mm}$. The only varying parameter is the maximal incident heat flux, from 2 to $10 \mathrm{MW} / \mathrm{m}^{2}$. The results are shown on figure 6 .

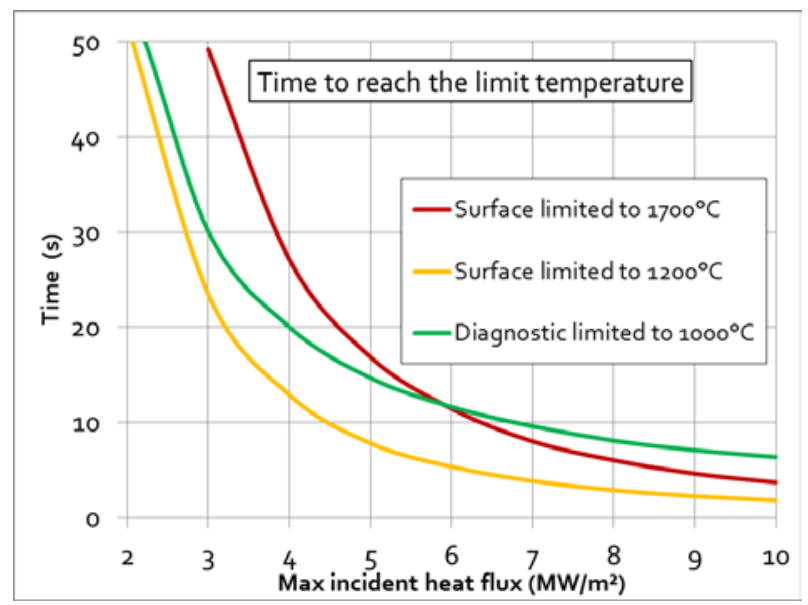

Figure 6: Time to reach the temperature limits for the PFU surface and embedded diagnostics

Obviously the time to reach $1200^{\circ} \mathrm{C}$ on the surface (yellow curve) is always lower than the time to reach $1700^{\circ} \mathrm{C}$ (red curve). First observation is that the PFU is not limited by the steel diagnostic (green curve) if the surface temperature is kept under $1200^{\circ} \mathrm{C}$, which is the temperature of the $\mathrm{W}$ carbidisation. However, if the surface is allowed to go up to $1700^{\circ} \mathrm{C}$, the diagnostics may become more limiting than the $\mathrm{W}$ coating. Indeed the maximal temperature is reached on the diagnostics before the surface reaches its own limit for incident heat flux lower than $6 \mathrm{MW} / \mathrm{m}^{2}$. For this kind of plasma parameters, the temperature of the embedded diagnostics should be checked carefully in addition to the surface temperature.

The most challenging part of the WEST project during the early phase of exploitation will be to reach incident heat fluxes of at least $10 \mathrm{MW} / \mathrm{m}^{2}$ during a time of at least $4 \mathrm{~s}$. Indeed during this phase several $\mathrm{W}$ monoblock PFUs will be installed on the lower divertor replacing some of the G PFU. The goal will be to test the $\mathrm{W}$-monoblocks at their nominal operation heat flux $\left(10 \mathrm{MW} / \mathrm{m}^{2}\right)$ in order to reach their thermal steady state conditions. Figure 7 shows the time to reach surface temperature of $1200^{\circ} \mathrm{C}$ (light color) and $1700^{\circ} \mathrm{C}$ (dark color) for 2 different decay lengths (2.5mm - red curve and $5.0 \mathrm{~mm}$ - blue curve). The time to reach the limit temperature is given as a function of the power absorbed by the divertor (roughly equals to the injected power minus the radiated power and the power conducted to the others components), while dashed lines give the correspondency between the absorbed power and the maximal incident heat flux onto the surface. The second 
reference scenario (called far X-point) is also included in this study. Its main characteristic is that the heat flux profile is much more peaked than for the close $\mathrm{X}$-point scenario by a factor of 3 . Therefore the area impacted with the highest heat flux is smaller with this scenario.

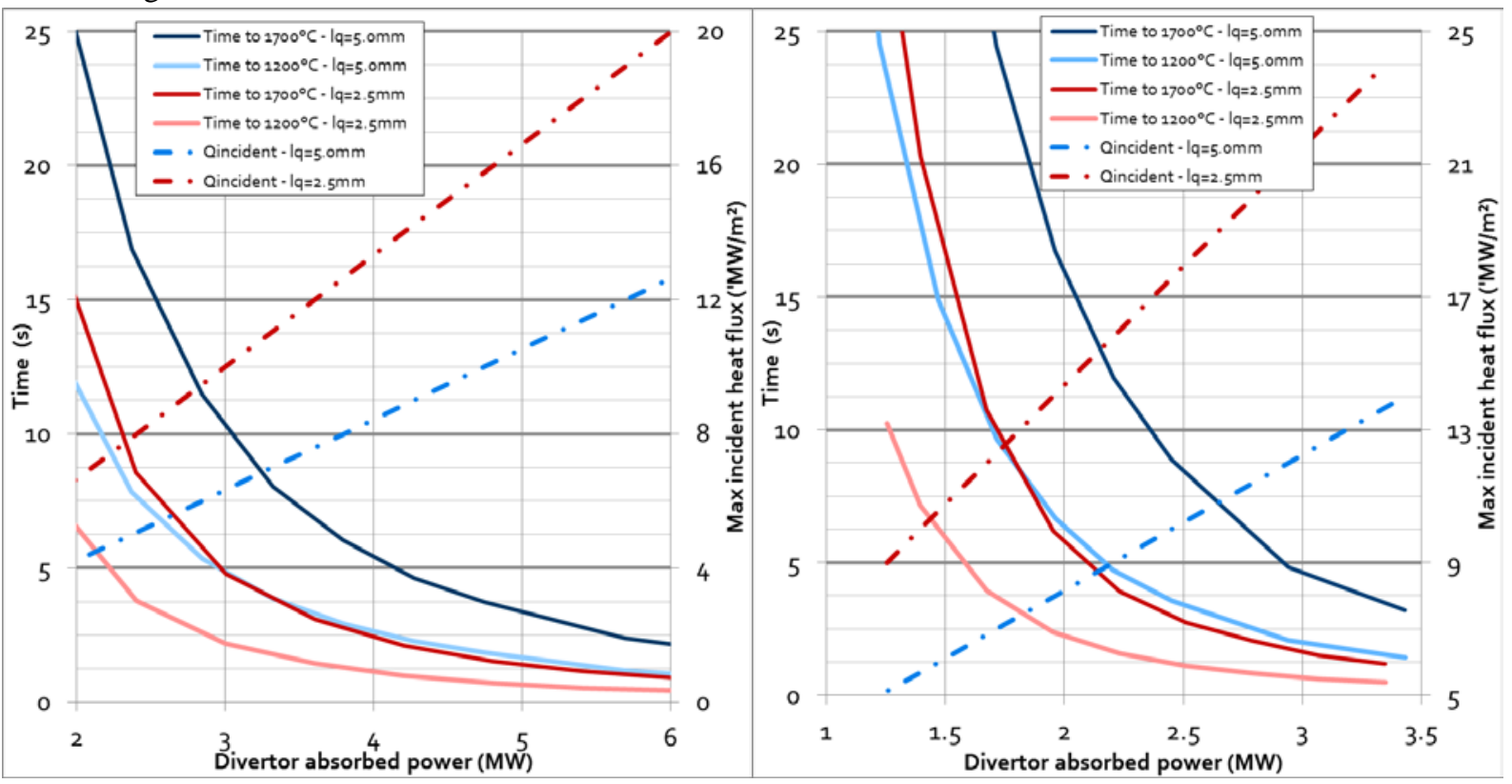

Figure 7: Time limits for the graphite PFU as a function of the divertor absorbed power - close X-point (left) and far X-point (right)

These graphs represent the following result: for $10 \mathrm{MW} / \mathrm{m}^{2}$ maximal incident heat flux on the G-PFU surface in the close X-point scenario, the absorbed power is 5MW (lq $5 \mathrm{~mm}$ ) or 3MW (lq 2.5mm). For this power, the time to reach $1200^{\circ} \mathrm{C} / 1700^{\circ} \mathrm{C}$ is $1.8 \mathrm{~s} / 3.7 \mathrm{~s}$ (lq $5 \mathrm{~mm}$ ) and $2.2 \mathrm{~s} / 4.8 \mathrm{~s}$ (lq $2.5 \mathrm{~mm}$ ). The main results are summarised in the next table.

\begin{tabular}{l|ccc|}
\cline { 2 - 4 } $\begin{array}{l}\left(\mathrm{l}_{\mathrm{q}} 2.5 \mathrm{~mm} /\right. \\
5.0 \mathrm{~mm})\end{array}$ & $\begin{array}{c}\text { P absorbed } \\
(\mathrm{MW})\end{array}$ & $\begin{array}{c}\text { Time to } \\
1200^{\circ} \mathrm{C}(\mathrm{s})\end{array}$ & $\begin{array}{c}\text { Time to } \\
1700^{\circ} \mathrm{C}(\mathrm{s})\end{array}$ \\
\hline Close X-point & $3 / 5$ & $2.2 / 1.8$ & $4.8 / 3.7$ \\
Far X-point & $1.4 / 2.5$ & $7.2 / 3.6$ & $20.3 / 8.9$
\end{tabular}

Table 2 : Divertor absorbed power and time to reach the limit of $1200^{\circ} \mathrm{C}$ and $1700^{\circ} \mathrm{C}$ for two scenarios and two decay lengths $(2.5 \mathrm{~mm} / 5.0 \mathrm{~mm})$

It can be concluded from these simulations that it is possible to heat the G-PFU during a time long enough (> 4s) that adjacent W monoblocks PFU reaches its steady state conditions. However, for the close Xpoint scenario, the temperature limit to be considered has to be $1700^{\circ} \mathrm{C}$, while for the far $\mathrm{X}$-point scenario the limit at $1200^{\circ} \mathrm{C}$ could be considered, enhancing the lifetime of the $\mathrm{W}$ coating.

\section{Conclusion and perspectives}

The results presented in this paper confirm the suitability of the graphite PFU including its attachment geometry for the required heat flux. It is also shown that the stainless steel based diagnostics embedded in some G-PFU should not limit the operation more than the W coating for the challenging part of the WEST research plan (high power plasma). However in the case of low incident heat fluxes (low power plasma), and if the surface is allowed to reach higher temperatures (long duration pulse), the diagnostics will be a limit to check. About the specific case of LP, it has been demonstrated by experiment that the instrumentation can reach higher temperature than expected. Consequently, it has been decided to change the support material of the probes from stainless steel to tantalum which is much more tolerant to high temperatures.

It is also shown that the thermal simulations exhibit a good agreement with the HHF experiment.

Finally, the last section demonstrates that there is a trade-off between the level of flux required $\left(10 \mathrm{MW} / \mathrm{m}^{2}\right.$ in this paper), the wetted area and the coating lifetime. But in any case, it seems possible to operate 
at a power so that the maximal incident heat flux on the lower divertor is $10 \mathrm{MW} / \mathrm{m}^{2}$ during a time longer than the time for the $\mathrm{W}$ monoblock PFU to reach its steady-state.

Some work has still to be done, in particular an HHF test on the graphite PFU including the W coating; the expected performances of the coating should be confirmed on the G-PFU itself.

\section{References}

[1] J. Bucalossi et al., Fusion Engineering and Design 89 (2014) 907-912.

[2] H. Greuner et al., Journal of Nuclear Materials 367-370 (2007) 1444.

[3] M. Firdaouss et al., Fusion Engineering and Design Available online 7 January 2015, ISSN 0920-3796,

http://dx.doi.org/10.1016/j.fusengdes.2014.12.024

[4] R. Neu et al., Physica Scripta T138 (2009) 014038

[5] C. Ruset et al., Fusion Engineering and Design 86 (2011) 1677-1680 